\title{
Beneficial Effects of Pioglitazone on Retardation of Persistent Atrial Fibrillation Progression in Diabetes Mellitus Patients
}

\author{
Bing LiU, ${ }^{1} \mathrm{MD}$, Jiancheng WANG,${ }^{2} \mathrm{MD}$, and Guoxing WANG,${ }^{1} \mathrm{MD}$
}

\begin{abstract}
SUMMARY
This study aimed to explore the effects of pioglitazone treatment on progression from persistent atrial fibrillation (AF) to permanent atrial fibrillation in diabetes mellitus (DM) patients and to investigate the possible mechanisms involved in those effects.

A total of 146 diabetes mellitus (DM) patients with firstly identified persistent AF were selected. Seventy patients were randomized into the pioglitazone ( $30 \mathrm{mg} /$ day) group and 76 into the placebo group. Pro-collagen type I carboxyterminal peptide (PICP), advanced glycation end products (AGEs), and angiotensin II were assayed and left atrial diameter (LA diameter) was measured at the first presence of persistent AF, and at 6 and 14 months of follow-up. The time point of identification of permanent $\mathrm{AF}$ and the incidence of permanent $\mathrm{AF}$ in the patients were all recorded.

Thirty-seven (49\%) of the 76 patients in the placebo group and $21(30 \%)$ of the 70 patients in the pioglitazone group progressed to permanent $\mathrm{AF}(P=0.028)$. No significant differences existed in the follow-up time $(20.5 \pm 3.97$ months for pioglitazone group versus $20.9 \pm 4.14$ months for placebo group) between the two groups $(P=0.535)$. In the pioglitazone group, no significant change was found in angiotensin II level. The PICP level did not change significantly at 6-months of follow-up, but decreased significantly at 14-months of follow-up $(P=0.032)$. The AGE $(P=0.037$ at 6-month follow-up, $P<0.035$ at 14-month follow-up) level was significantly lower at both 6 and 14-months of followup.

By lowering the PICP level, pioglitazone treatment may decrease the incidence of permanent AF in DM patients with persistent AF, which may be associated with the suppressing effect of pioglitazone on AGEs. (Int Heart J 2014; 55:
\end{abstract} 499-505)

Key words: Type I carboxy-terminal peptide, Left atrial diameter, Atrial dilation, Angiotensin II, Advanced glycation end products, Atrial fibrosis, Atrial structural remodeling, Cardioversion, Arrhythmia, Clinical trial

$\mathrm{M}$ any pathophysiologic mechanisms are involved in the progression from persistent to permanent atrial fibrillation (AF) in diabetes mellitus (DM) patients. ${ }^{1)}$ Among them, atrial fibrosis has been proven to be an important mechanism. Fibrosis can induce atrial fractionated potentials and conduction delay by accelerating the formation of anchoring points for reentry circuits and the forward propagation of wavelets. Collagen fibers play an important role in atrial fibrosis and atrial structural remodeling. ${ }^{2)}$ During synthesis of the fibril forming collagen type I in atria, the serum procollagen type I carboxy-terminal peptide (PICP) is cleaved from the pro-collagen and can be detected in the blood. A previous study has linked serum concentrations of PICP to the prevalence or incidence of atrial fibrillation (AF).

By suppressing the production of collagen fibers and promoting their degradation, the newly developed upstream therapy (statin and renin-angiotensin system inhibitors) has become a promising strategy to prevent AF progression. ${ }^{4-6)}$ Nevertheless, the effects of upstream therapy are still controversial.
Some studies did not find any effects of upstream therapies by renin-angiotensin system inhibitors or statins on AF recurrence. ${ }^{7)}$

In clinical practice, pioglitazone has become a standard therapy for DM. By activating peroxisome proliferator-activated receptor-gamma (PPAR-gamma), pioglitazone acquires several pleiotropic properties such as inhibiting inflammatory and oxidant processes, ${ }^{8-12)}$ which may contribute to attenuating atrial fibrosis in the setting of AF. Therefore, it has attracted much attention as a new upstream therapy in recent studies on $\mathrm{DM}$ patients with $\mathrm{AF}$. Gu, et al have indicated that the chance of success in catheter ablation can be significantly enhanced by pioglitazone in DM patients with paroxysmal $\mathrm{AF}^{13)}$ However, whether the pioglitazone treatment is beneficial to persistent AF patients in AF progression has not yet been investigated.

Thus, in the present study, we attempted to further examine the controversial upstream therapy by exploring the effects of pioglitazone treatment on progression from persistent to

From the Departments of ${ }^{1}$ Emergency and ${ }^{2}$ Clinical Laboratory, Beijing Friendship Hospital, Capital Medical University, Beijing, China

Addres for correspondence: Bing Liu, MD, Department of Emergency, Beijing Friendship Hospital, Capital Medical University, Beijing 100050, China. E-mail: liubing3207508@163.com

Received for publication April 6, 2014. Revised and accepted May 16, 2014.

Released in advance online on J-STAGE October 14, 2014.

All rights reserved by the International Heart Journal Association. 
permanent $\mathrm{AF}$ in $\mathrm{DM}$ patients and investigating the possible mechanisms and implications underlying these effects.

\section{MeTHODS}

Study population: This was a prospective, randomized, placebo-controlled, double-blind trial carried out in Beijing Friendship Hospital Affiliated to the Capital Medical University. Inclusion criteria were a history of DM and the first presence of persistent AF defined as AF lasting beyond 7 days or demanding electrical or chemical cardioversion for termination. ${ }^{14)} \mathrm{Ex}$ clusion criteria were liver or renal dysfunction; history of hyperthyroidism, pulmonary fibrosis, cirrhosis or scleroderma; current treatment with pioglitazone, an angiotensin converting enzyme inhibitor (ACEI), or angiotensin II receptor blocker (ARB); history of acute coronary syndrome (ACS); congestive heart failure; pregnancy; or history of catheter ablation. From February 2012 to July 2013, a total of 264 consecutive DM patients with firstly identified persistent AF were initially referred to our study. Among these 264 patients, 118 were excluded due to current treatment with ACEI/ARB (50 patients), fibrosis disease (18 patients) such as pulmonary fibrosis and cirrhosis, history of acute coronary syndrome (15 patients), and contraindications to pioglitazone (35 patients) including liver dysfunction, renal dysfunction, and congestive heart failure. According to a computer generated randomization list, 146 qualified patients were assigned to treatment with placebo or pioglitazone $(30 \mathrm{mg} /$ day) at the first presence of persistent AF. All pills were identical with the same color, size and taste to ensure blindness. During the follow-up, ACEI and ARB were not prescribed to patients. The physicians participating in this study were blinded to the randomized assignment of the patients. The institutional review board of our institution reviewed and approved the study protocol and informed consent forms.

Blood analysis: At the first presence of persistent $\mathrm{AF}$ and at 6 and 14-months follow-up, peripheral venous samples were obtained in the morning after overnight fasting. The serum was extracted from these samples and stored at $-80^{\circ} \mathrm{C}$. PICP level was measured by enzyme linked immunosorbent assay using commercial antisera (Jianchen Biological Institution, China) according to the manufacturers' protocol. ${ }^{15)}$ Angiotensin II was measured by radioimmunoassay ${ }^{16)}$ using a kit obtained from Abcam (HK) Ltd. Using Munch's method, ${ }^{17)}$ fluorescence was used to evaluate advance glycation end products (AGEs) which were expressed as arbitrary units (a.u.). All measures were assessed in duplicate.

Echocardiography analysis: A 2-dimensional echocardiogram was performed to evaluate left ventricular ejection fraction (LVEF) and left atrial diameter (LA diameter) at the first presence of persistent AF, and at 6 and 14-months of follow-up. The analysis was carried out by 2 observers without any knowledge of the clinical data.

Follow-up: Patients were allowed to leave the hospital when sinus rhythm (SR) was sustained for 2 days. All patients were checked every other month thereafter. At each visit, blood pressure, a resting 12-lead ECG, and history of arrhythmia-related symptoms were recorded, and 24-hour Holter monitoring was carried out every month to detect asymptomatic AF. ECG and arrhythmia specialists were always available when any episode of palpitation occurred in our patients. After the confir- mation of recurrent AF by 12-lead ECG, a continuous infusion of amiodarone was introduced until stable SR was obtained. Electrical cardioversion was conducted when unstable haemodynamics emerged or amiodarone was unable to convert the AF. When stable SR could be maintained in the follow-up, the amiodarone was discontinued. Failed attempts of electrical cardioversion and a medical rhythm control approach to restore sinus rhythm were considered as progression to permanent AF. ${ }^{14,18)}$

Endpoints: The primary endpoint of this study was the incidence of progression to permanent AF. The secondary endpoint was to identify the significantly changed variables and the correlation of such changes with the incidence of permanent AF.

Statistical analysis: The estimated sample size was based on our estimate of the primary endpoint difference that the incidence of permanent AF is to be lower by $25 \%$ in the pioglitazone group than in the placebo group. ${ }^{13)}$ With a significance level of 0.05 , a total of at least 140 randomized patients (70 in each arm) provided the study with $85 \%$ power. Continuous variables are expressed as the mean $\pm \mathrm{SD}$, and categorical variables as a percentage. Continuous variables were analyzed for a normal distribution with the Kolmogorov-Smirnov test. Differences in paired variables between baseline, and 6 and 14-months of follow-up were compared with the paired- $t$ test. The independent-samples $t$ test was used to compare continuous variables between the pioglitazone and placebo groups; otherwise, the chi-square test was applied. Statistical significance was assumed at $P<0.05$. Pearson correlation coefficients were used to evaluate the correlation of atrial fibrosis with various parameters. The time to permanent AF was analyzed with Kaplan-Meier statistics and compared with the logrank test.

\section{Results}

Study population: Table I presents the baseline clinical variables of the study population. No differences were found in age, gender, medical therapy, or history of smoking, drinking, coronary artery disease, and valvular heart disease between the placebo and pioglitazone groups. There were also no differences between the study groups with respect to lipid level, $\mathrm{HbA1c}$, systolic blood pressure (SBP), diastolic blood pressure (DBP), LVEF, LA diameter, or serum PICP, AGEs and angiotensin II levels. All patients were followed-up for at least 14 months (range, 14-30 months). The safety of pioglitazone was reliable in general. In our study, mild peripheral edema occurred in 6 patients from the pioglitazone group and in 3 patients from the control group $(P=0.312)$. No acute heart failure was found in any patient.

Primary endpoint: The number of patients that suffered from recurrent $\mathrm{AF}$ was $48(63.2 \%)$ in the placebo and $31(44.3 \%)$ in the pioglitazone group $(P=0.030)$. There were $6 \mathrm{AF}$ episodes detected by Holter monitoring in the placebo group and 4 in the pioglitazone group. The primary outcome of progression to permanent AF occurred in 37 of the 76 placebo group patients (49\%) and in 21 of the 70 pioglitazone group patients (30\%) $(P$ $=0.028)$. No significant difference existed in the follow-up time $(20.5 \pm 3.97$ months for pioglitazone group versus $20.9 \pm$ 4.14 months for placebo group) between the two groups $(P=$ 
Table I. Clinical Characteristics

\begin{tabular}{lccc}
\hline & Placebo $(n=76)$ & Pioglitazone $(n=70)$ & $P$ \\
\hline Male gender & $50(65.8 \%)$ & $52(74.3 \%)$ & 0.284 \\
Age (years) & $62.25 \pm 12.39$ & $60.70 \pm 12.25$ & 0.449 \\
Hypertension & $23(30.3 \%)$ & $20(28.6 \%)$ & 0.859 \\
Current smokers & $42(55.3 \%)$ & $41(58.6 \%)$ & 0.739 \\
Alcoholic history & $16(21.1 \%)$ & $19(27.1 \%)$ & 0.441 \\
CAD & $23(30.3 \%)$ & $20(28.6 \%)$ & 0.857 \\
VHD & $7(9.2 \%)$ & $5(7.1 \%)$ & 0.767 \\
CHOL (mmol/L) & $5.44 \pm 1.61$ & $5.30 \pm 0.97$ & 0.541 \\
TG (mmol/L) & $2.24 \pm 1.77$ & $2.42 \pm 1.65$ & 0.615 \\
LDL-C (mmol/L) & $2.69 \pm 0.73$ & $2.72 \pm 0.46$ & 0.706 \\
HDL-C (mmol/L) & $1.08 \pm 0.38$ & $1.10 \pm 0.24$ & 0.732 \\
Amiodarone & $15(19.7 \%)$ & $13(17.1 \%)$ & 0.831 \\
Beta-blockers & $29(38.2 \%)$ & $29(41.4 \%)$ & 0.736 \\
CCB & $13(17.1 \%)$ & $14(20 \%)$ & 0.675 \\
Propafenone & $10(13.2 \%)$ & $6(8.6 \%)$ & 0.435 \\
Statins & $26(34.2 \%)$ & $22(31.4 \%)$ & 0.729 \\
LVEF & $0.57 \pm 0.04$ & $0.58 \pm 0.04$ & 0.494 \\
HbAlc (\%) & $6.19 \pm 1.45$ & $6.41 \pm 1.83$ & 0.407 \\
SBP & $132 \pm 19$ & $131 \pm 21$ & 0.877 \\
DBP & $76 \pm 10$ & $77 \pm 12$ & 0.653 \\
\hline
\end{tabular}

CHOL indicates cholesterol; TG, triglycerides; LDL-C, low density lipoprotein-cholesterol; HDL, high density lipoprotein-cholesterol; LVEF, left ventricular ejection fraction; $\mathrm{CCB}$, calcium channel blocker; $\mathrm{CAD}$, coronary artery disease; VHD, valvular heart disease; HbAlc, haemoglobin A1c; SBP, systolic blood pressure; and DBP, diastolic blood pressure.

\section{Freedom plot from permanent AF}

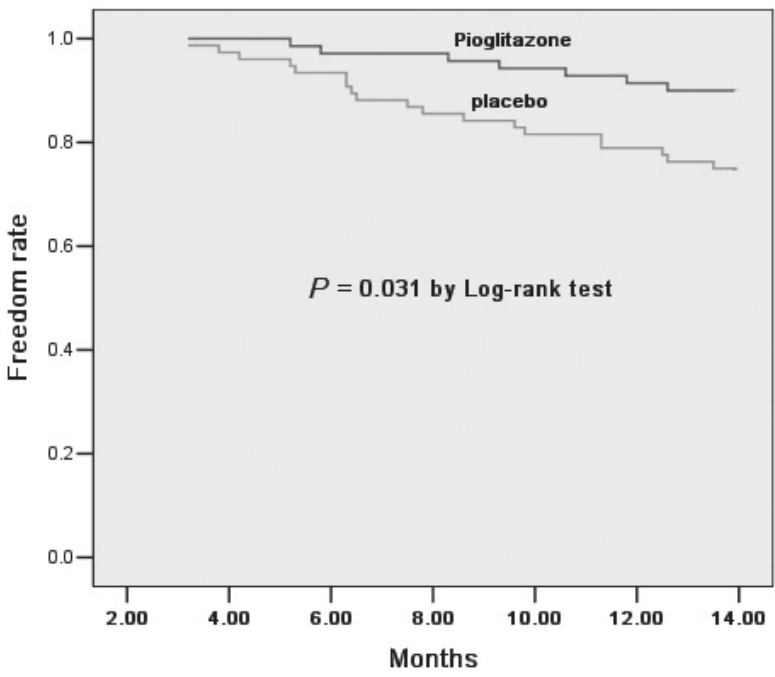

Figure 1. Kaplan-Meier permanent AF-free survival curve for pioglitazone and placebo groups. AF indicates atrial fibrillation.

0.535). Kaplan-Meier permanent AF-free survival analysis for 14 months follow-up is presented in Figure 1. Pioglitazone significantly decreased the incidence of permanent $\mathrm{AF}(P=$ 0.031).

Secondary endpoint: The patients that progressed to permanent AF during the 14 months of follow-up demonstrated a higher baseline LA diameter $(P<0.01)$, PICP $(P<0.01)$, AGE $(P<0.01)$, and angiotensin II $(P<0.01)$ levels than patients free from permanent AF (Figure 2). The lipid level, SBP, DBP, LVEF, and LA diameter did not change in either group. The HbA1c $(P=0.031)$ significantly decreased in the pioglitazone
$\operatorname{PICP}(n g / m L)$
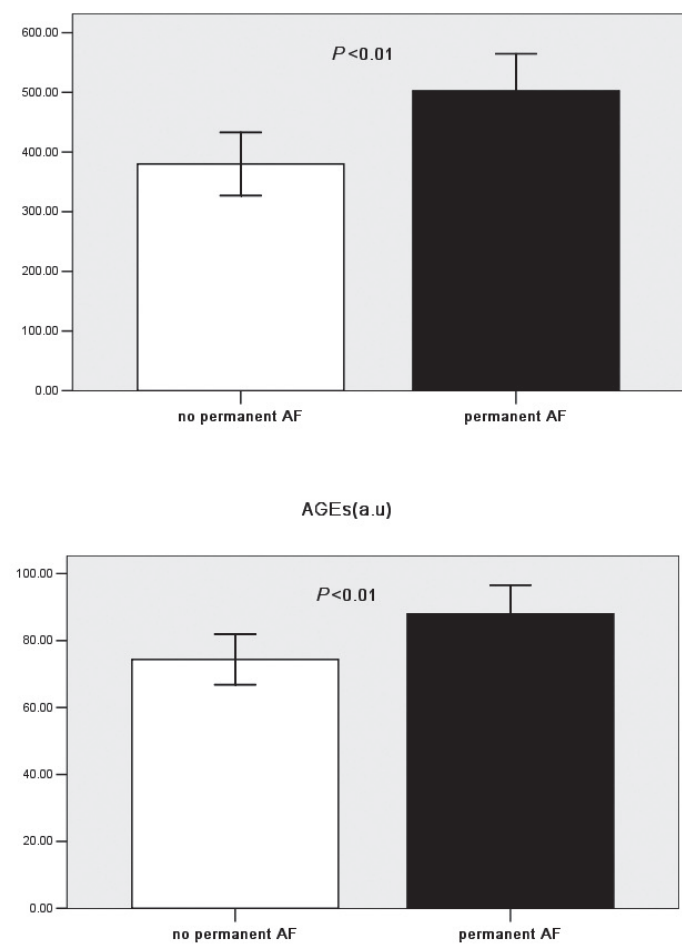

Angiotensin II(pg/dL)

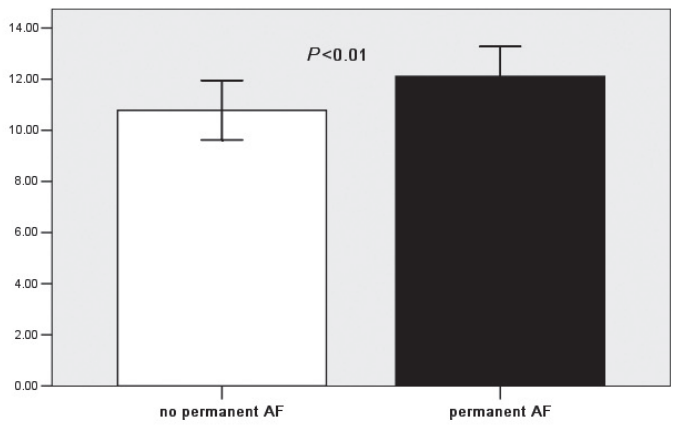

LA diameter(mm)

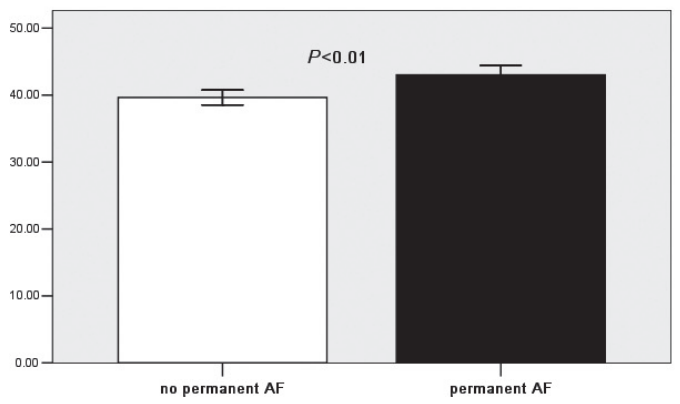

Figure 2. Baseline mean serum PICP levels, serum AGEs levels, serum angiotensin II levels, and LA diameters in patients who did not develop permanent AF during follow-up (white bars) and in patients who developed permanent AF during follow-up (black bars). AF indicates atrial fibrillation; LA diameter, left atrial diameter; PICP, pro-collagen type I carboxy-terminal peptide; and AGEs, advanced glycation end products. 
Table II. Variables at Baseline and at Month 14

\begin{tabular}{lcccccc}
\hline & \multicolumn{3}{c}{ Placebo group $(n=76)$} & \multicolumn{3}{c}{ Pioglitazone group $(n=70)$} \\
& Month 0 & Month 14 & $P$ & Month 0 & Month 14 & $P$ \\
\hline CHOL (mmol/L) & $5.44 \pm 1.61$ & $5.41 \pm 1.33$ & 0.677 & $5.30 \pm 0.97$ & $5.33 \pm 0.96$ & 0.388 \\
TG $(\mathrm{mmol} / \mathrm{L})$ & $2.24 \pm 1.77$ & $2.19 \pm 1.56$ & 0.438 & $2.42 \pm 1.65$ & $2.41 \pm 1.62$ & 0.377 \\
LDL-C (mmol/L) & $2.69 \pm 0.73$ & $2.73 \pm 0.84$ & 0.356 & $2.72 \pm 0.46$ & $2.73 \pm 0.47$ & 0.686 \\
HDL-C (mmol/L) & $1.08 \pm 0.38$ & $1.06 \pm 0.35$ & 0.252 & $1.10 \pm 0.24$ & $1.08 \pm 0.24$ & 0.216 \\
LVEF & $0.57 \pm 0.04$ & $0.57 \pm 0.04$ & 0.862 & $0.58 \pm 0.04$ & $0.57 \pm 0.05$ & 0.429 \\
HbA1c (\%) & $6.19 \pm 1.45$ & $6.21 \pm 1.54$ & 0.744 & $6.41 \pm 1.83$ & $6.07 \pm 2.26$ & $0.031 *$ \\
SBP & $132 \pm 19$ & $133 \pm 18$ & 0.612 & $131 \pm 21$ & $132 \pm 17$ & 0.750 \\
DBP & $76 \pm 10$ & $75 \pm 12$ & 0.447 & $77 \pm 12$ & $76 \pm 12$ & 0.431 \\
\hline
\end{tabular}

CHOL indicates cholesterol; TG, triglycerides; LDL-C, low density lipoprotein-cholesterol; HDL, high density lipoproteincholesterol; LVEF, left ventricular ejection fraction; HbAlc, hemoglobin A1c; SBP, systolic blood pressure; and DBP, diastolic blood pressure. * Difference is significant.
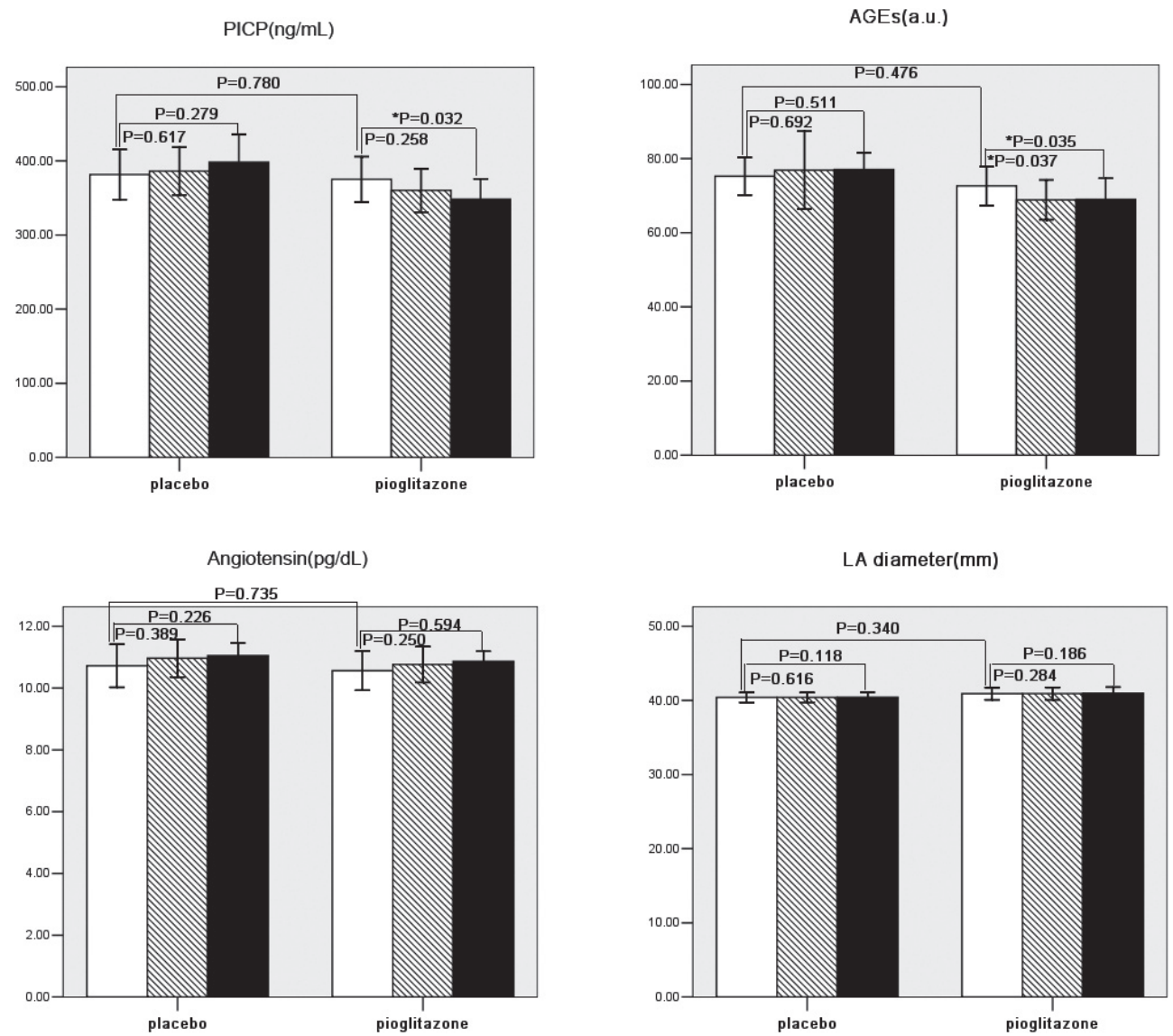

Figure 3. Baseline (white bars), 6 month (stripe bars), and 14 month (black bars) mean serum PICP levels, serum angiotensin II levels, LA diameters, and serum AGEs levels in patients randomized to the placebo-treatment group and in patients randomized to the pioglitazone-treatment group. AF indicates atrial fibrillation; LA diameter, left atrial diameter; PICP, pro-collagen type I carboxy-terminal peptide; and AGEs, advanced glycation end products. * Difference is significant.

group after 14 months (Table II). In the placebo group, the serum PICP, AGE and angiotensin II levels remained stable during the 14-month follow-up. In the pioglitazone group, no significant change was found in angiotensin II level. The PICP did not change significantly at 6-months of follow-up, but decreased significantly at 14 -months of follow-up $(P=0.032)$. The $\operatorname{AGE}(P=0.037$ at 6 -month follow-up, $P<0.035$ at 14-month follow-up) level had decreased significantly at both
6 and 14 months of follow-up (Figure 3).

We examined the correlation of the change in PICP with the change in AGEs after 14-months of follow-up in the pioglitazone group and found that the change in PICP was significantly correlated with the change in AGEs $(r=0.567, P<$ 0.01). The correlation of the change in AGEs with the change in HbA1c was also calculated. The results showed that the change in AGEs was positively correlated with the change in 

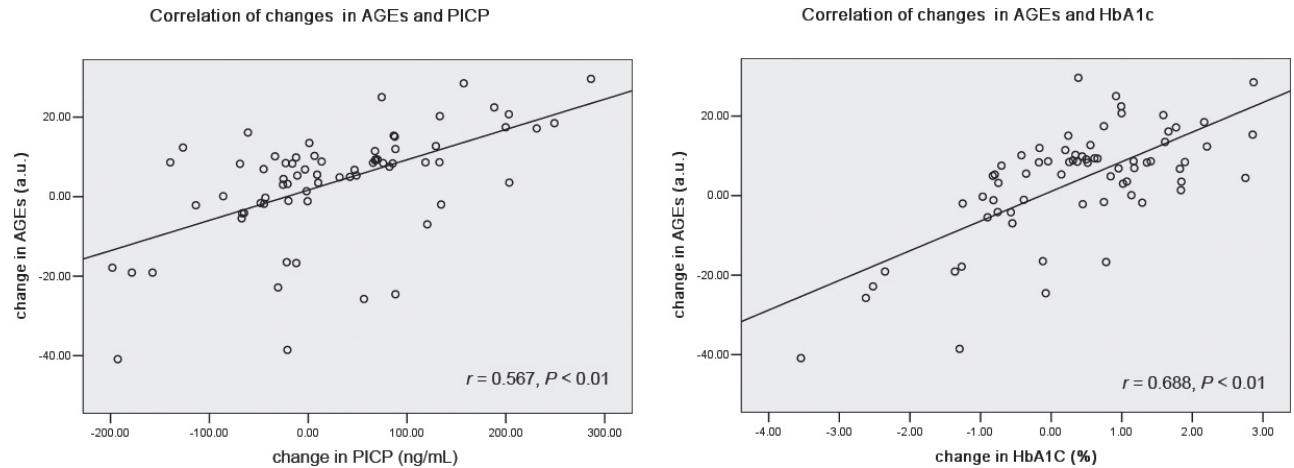

Figure 4. Correlation of the change in AGEs with the changes in HbAlc and PICP after 14-months of follow-up. PICP indicates pro-collagen type I carboxy-terminal peptide; AGEs, advanced glycation end products; and HbAlc, Haemoglobin A1c.

$\operatorname{HbA1c}(r=0.688, P<0.01)$ (Figure 4).

\section{DiscuSSION}

Atrial fibrosis has been observed in AF patients and proved to be an important mechanism for $\mathrm{AF}$ progression in experimental models. ${ }^{19-22)}$ It has been found to expand with age in animal models and humans. ${ }^{23,24)}$ Although many mechanisms such as a reduction of conduction velocity, heterogeneity of the conduction tissue, and reentrant mechanisms have been proposed for $\mathrm{AF}^{25-27)}$ the mechanism of $\mathrm{AF}$ induced by atrial fibrosis is still under debate. Microscopic cardiac biopsy is the most reliable method to measure the extent of atrial fibrosis, but it cannot be applied on a wide scale because of its invasive nature. Therefore, some noninvasive serum markers such as PICP have gradually gained favor in clinical studies to assess atrial fibrosis. ${ }^{28-30)}$ Type I collagen is the major fibrillar collagen in atria. ${ }^{31-33)}$ During its synthesis, PICP is separated from procollagen I and released into blood. By measuring PICP, we may indirectly evaluate the extent of atrial collagen synthesis. ${ }^{34)}$ Some studies have shown that the serum PICP level and LA diameter might indirectly demonstrate the extent of left atrial fibrosis and predict post-operative AF even in patients without a history of $\mathrm{AF}^{3)}$ Our results confirmed these findings by demonstrating a higher baseline PICP level and LA diameter in patients that progressed to permanent AF.

AGEs are crystallized proteins which accumulate with aging. ${ }^{35)}$ Non-enzymatic reactions between proteins and sugar residues produce them, and the process is markedly accelerated in the setting of $\mathrm{DM}^{36)}$ AGEs can directly improve protein cross-linking including the synthesis of type I collagen and elastin in the extracellular matrix. In addition, when AGEs interact with AGE receptors (RAGE), the production of matrix metalloproteinases (MMPs) and tissue growth factor-beta were stimulated. ${ }^{37-39)}$ All of these actions together lead to atrial fibrosis, resulting in multiple wavelets and reentrant anchor points in atria. A recent clinical study also found that the serum AGE level was higher in AF patients with or without diabetes mellitus. ${ }^{40)}$ Another important factor involved in atrial fibrosis is angiotensin II. Some studies have indicated that angiotensin II played an important role in atrial fibrosis in experimental models. ${ }^{41)}$ By promoting tissue growth factor expression, fibroblast proliferation, trans-differentiation, and the production of colla- gen I and angiotensin II significantly facilitated the development and progression of atrial fibrosis. ${ }^{42,43)}$ It was also demonstrated that blockade of angiotensin II with an ACEI and/or $\mathrm{ARB}$ retarded the progression of atrial fibrosis and $\mathrm{AF}^{44,45)}$ In present study, we observed higher baseline angiotensin II and AGE levels in patients who had progressed to permanent AF. Furthermore, in the pioglitazone group, a significantly decreased AGE level was identified at 6-months of follow-up, and this occurred prior to the significant elevation of PICP level. Moreover, the change in PICP after 14-months of follow-up was positively correlated with the change in AGEs. All of the findings in our study further confirmed the pathophysiologic roles that angiotensin II and AGEs may play in atrial fibrosis.

Some studies have indicated that pioglitazone prevented age-related atrial structure remodeling in rat models ${ }^{46)}$ and attenuated atria fibrosis in rabbits with congestive heart failure. ${ }^{47)}$ Consistently, our present study observed significantly decreased PICP levels in the pioglitazone group at 14-months of follow-up. However, no significant change in LA diameter was observed in our study. We speculate that the left atrial dilation caused by atrial fibrosis is a lengthy process and can not be detected during 14 months of follow-up. The mechanisms underlying the inhibitory effects of pioglitazone on atrial fibrosis are uncertain. Anti-inflammation, ${ }^{48)}$ anti-oxidation ${ }^{49)}$ and inhibiting angiotensin II, AGEs and TGF cascade, ${ }^{50)}$ were all proposed as possible mechanisms of this process. Among them, our study mainly focused on two possible therapeutic targets revealed in recent experimental studies of pioglitazone: angiotensin II and AGEs.

A growing body of evidence suggests that pioglitazone might modulate AGEs-AGE receptor (RAGE) axis by inhibiting the effects of AGEs, increasing soluble RAGE, and downregulating RAGE expression. In this regard, the adverse effects of AGEs on the pancreatic beta cell can be inhibited by pioglitazone. ${ }^{51)}$ Another study indicated that pioglitazone significantly increased the soluble RAGE level in patients with DM. ${ }^{52}$ Furthermore, oral pioglitazone treatment in hypercholesterolemic rabbits caused significant alleviation of aortic valve calcification by targeted reduction of RAGE activation. ${ }^{53)}$ Nevertheless, the effect of pioglitazone on serum AGE levels has seldom been investigated in a clinical study. By showing a significantly decreased AGE level in the pioglitazone group at 6 and 14-months of follow-up, our study results indicated that pioglitazone may suppress serum AGEs in DM patients with 
AF. We also found that the significant decrease in AGEs was positively correlated with the decrease in $\mathrm{HbAlc}$ in the pioglitazone group, implying that the antidiabetic effects of pioglitazone may be associated with its inhibition of AGE levels.

Many clinical and experimental studies have reported that pioglitazone inhibited the effect and decreased the level of angiotensin II. Saiki, et $a l^{54)}$ showed that pioglitazone treatment for 16 weeks resulted in a decrease in plasma angiotensin II in DM patients. Subramanian, et $a l^{55)}$ found that pioglitazone retarded angiotensin II-related atherosclerosis progression by activating the PPAR-gamma receptors in smooth muscle cells. Furthermore, pioglitazone suppressed angiotensin II-induced atrial fibrosis in an experimental model. ${ }^{56)}$ However, in our clinical study, we did not find that the angiotensin II level was significantly decreased in the pioglitazone group. We speculate that the volatility of the angiotensin II level in DM patients, which was influenced by many factors that were not fully assessed in our study such as blood pressure, serum sodium concentration, blood volume and autonomic nervous system, may account for the inconsistency with previous studies.

A recent cohort study indicated that pioglitazone prevented DM patients from incipient $\mathrm{AF}^{57)}$ and $\mathrm{Gu}$, et al reported that pioglitazone ( $30 \mathrm{mg} /$ day) decreased the incidence of recurrent $\mathrm{AF}$ and reablation in AF patients who underwent ablation. ${ }^{13)}$ Similar to these observations, we found that the pioglitazone group demonstrated a significantly lower incidence of recurrent $\mathrm{AF}$, and more importantly, less recurrent $\mathrm{AF}$ progressed to permanent $\mathrm{AF}$ in the pioglitazone group compared with the placebo group. These findings, together with the suppressing effect of pioglitazone on AGEs and the close correlation between atrial fibrosis and AGEs observed in our study, suggest that the beneficial effects of pioglitazone on retardation of persistent AF progression in DM patients is associated with the inhibitory effects of pioglitazone on serum AGE levels.

Study limitations: First, although accelerated by AF and DM, atrial fibrosis is still a lengthy process. Therefore, the 14 months of follow-up might be too short to show the effects of pioglitazone on atrial structure remodeling such as left atrial dilation. Second, AGEs contain not only fluorescent AGEs but also non-fluorescent AGEs. Since non-fluorescent AGEs were not assayed in the present study, the association between nonfluorescent AGEs and atrial fibrosis was not investigated. Third, as the study enrolled a small number of patients from a single center, a larger randomized trial conducted in multiple centers is needed to consolidate our findings. Finally, pioglitazone possesses several pleiotropic properties, and many other mechanisms of pioglitazone for preventing AF progression such as anti-inflammation were not investigated in our study.

Conclusion: In conclusion, this study shows pioglitazone treatment may decrease the incidence of permanent AF in DM patients with persistent AF by lowering PICP levels, which may be correlated with the inhibitory effects of pioglitazone on AGEs. These findings may expand the AF prevention mechanisms of pioglitazone and improve the outcome of DM patients with persistent $\mathrm{AF}$.

\section{ReFERENCE}

1. Lip GY, Varughese GI. Diabetes mellitus and atrial fibrillation: perspectives on epidemiological and pathophysiological links. Int
J Cardiol 2005; 105: 319-21.

2. Kato T, Yamashita T, Sekiguchi A, et al. What are arrhythmogenic substrates in diabetic rat atria? J Cardiovasc Electrophysiol 2006; 17: 890-4.

3. Swartz MF, Fink GW, Sarwar MF, et al. Elevated pre-operative serum peptides for collagen I and III synthesis result in post-surgical atrial fibrillation. J Am Coll Cardiol 2012; 60: 1799-806.

4. Goette A. Upstream therapy for atrial fibrillation in heart failure. Heart Fail Clin 2013; 9: 417-25. (Review)

5. Tziakas DN, Chalikais GK, Stakos DA, et al. Effect of angiotensin-converting enzyme insertion/deletion genotype on collagen type I synthesis and degradation in patients with atrial fibrillation and arterial hypertension. Expert Opin Pharmacother 2007; 8: 2225-34.

6. Loffredo L, Angelico F, Perri L, Violi F. Upstream therapy with statin and recurrence of atrial fibrillation after electrical cardioversion. Review of the literature and meta-analysis. BMC Cardiovasc Disord 2012; 12: 107. (Review)

7. Savelieva I, Kakouros N, Kourliouros A, Camm AJ. Upstream therapies for management of atrial fibrillation: review of clinical evidence and implications for European Society of Cardiology guidelines. Part II: secondary prevention. Europace 2011; 13: 61025. (Review)

8. Shimano M, Tsuji Y, Inden Y, et al. Pioglitazone, a peroxisome proliferator-activated receptor-gamma activator, attenuates atrial fibrosis and atrial fibrillation promotion in rabbits with congestive heart failure. Heart Rhythm 2008; 5: 451-9.

9. Kume O, Takahashi $\mathrm{N}$, Wakisaka $\mathrm{O}$, et al. Pioglitazone attenuates inflammatory atrial fibrosis and vulnerability to atrial fibrillation induced by pressure overload in rats. Heart Rhythm 2011; 8: 27885.

10. Vaiopoulos AG, Marinou K, Christodoulides C, Koutsilieris M. The role of adiponectin in human vascular physiology. Int J Cardiol 2012; 155: 188-93. (Review)

11. Li G, Liu T. The therapeutic strategies of enhancing adiponectin and lowering leptin may be benefit to controlling atrial fibrillation. Med Hypotheses 2009; 73: 122.

12. Xu D, Murakoshi N, Igarashi M, et al. PPAR- $\gamma$ activator pioglitazone prevents age-related atrial fibrillation susceptibility by improving antioxidant capacity and reducing apoptosis in a rat model. J Cardiovasc Electrophysiol 2012; 23: 209-17.

13. Gu J, Liu X, Wang X, et al. Beneficial effect of pioglitazone on the outcome of catheter ablation in patients with paroxysmal atrial fibrillation and type 2 diabetes mellitus. Europace 2011; 13: 125661.

14. Fuster V, Rydén LE, Cannom DS, et al. ACC/AHA/ESC 2006 Guidelines for the Management of Patients with Atrial Fibrillation: a report of the American College of Cardiology/American Heart Association Task Force on Practice Guidelines and the European Society of Cardiology Committee for Practice Guidelines (Writing Committee to Revise the 2001 Guidelines for the Management of Patients With Atrial Fibrillation): developed in collaboration with the European Heart Rhythm Association and the Heart Rhythm Society. Circulation 2006; 114: e257-354.

15. Martos R, Baugh J, Ledwidge M, et al. Diastolic heart failure: evidence of increased myocardial collagen turnover linked to diastolic dysfunction. Circulation 2007; 115: 888-95.

16. Kobayashi $\mathrm{H}$, Shimamoto K, Moriguchi O. Radioimmunoassay of human plasma angiotensin II (author's transl). Horumon To Rinsho 1979; 27: 665-9. (Japanese)

17. Münch G, Keis R, Wessels A, et al. Determination of advanced glycation end products in serum by fluorescence spectroscopy and competitive ELISA. Eur J Clin Chem Clin Biochem 1997; 35: 669-77.

18. Jahangir A, Lee V, Friedman PA, et al. Long-term progression and outcomes with aging in patients with lone atrial fibrillation: a 30year follow-up study. Circulation 2007; 115: 3050-6.

19. Spach MS, Boineau JP. Microfibrosis produces electrical load variations due to loss of side-to-side cell connections: a major mechanism of structural heart disease arrhythmias. Pacing Clin Electro- 
physiol 1997; 20: 397-413. (Review)

20. Spach MS, Josephson ME. Initiating reentry: the role of nonuniform anisotropy in small circuits. J Cardiovasc Electrophysiol 1994; 5: 182-209. (Review)

21. Frustaci A, Chimenti C, Bellocci F, Morgante E, Russo MA, Maseri A. Histological substrate of atrial biopsies in patients with lone atrial fibrillation. Circulation 1997; 96: 1180-4.

22. Kostin S, Klein G, Szalay Z, Hein S, Bauer EP, Schaper J. Structural correlate of atrial fibrillation in human patients. Cardiovasc Res 2002; 54: 361-79.

23. Anyukhovsky EP, Sosunov EA, Plotnikov A, et al. Cellular electrophysiologic properties of old canine atria provide a substrate for arrhythmogenesis. Cardiovasc Res 2002; 54: 462-9.

24. Hayashi H, Wang C, Miyauchi Y, et al. Aging-related increase to inducible atrial fibrillation in the rat model. J Cardiovasc Electrophysiol 2002; 13: 801-8.

25. Haïssaguerre M, Jaïs P, Shah DC, et al. Spontaneous initiation of atrial fibrillation by ectopic beats originatingin the pulmonary veins. N Engl J Med 1998; 339: 659-66.

26. Mandapati R, Skanes A, Chen J, Berenfeld O, Jalife J. Stable microreentrant sources as a mechanism of atrial fibrillation in the isolated sheep heart. Circulation 2000; 101: 194-9.

27. Skanes AC, Mandapati R, Berenfeld O, Davidenko JM, Jalife J. Spatiotemporal periodicity during atrial fibrillation in the isolated sheep heart. Circulation 1998; 98: 1236-48.

28. Kallergis EM, Manios EG, Kanoupakis EM, et al. Extracellular matrix alterations in patients with paroxysmal and persistent atrial fibrillation: biochemical assessment of collagen type-I turnover. J Am Coll Cardiol 2008; 52: 211-5.

29. Tziakas DN, Chalikias GK, Papanas N, et al. Circulating levels of collagen type I degradation marker depend on the type of atrial fibrillation. Europace 2007; 9: 589-96.

30. Sezai A, Hata M, Niino T, et al. Study of the factors related to atrial fibrillation after coronary artery bypass grafting: a search for a marker to predict the occurrence of atrial fibrillation before surgical intervention. J Thorac Cardiovasc Surg 2009; 137: 895-900.

31. Boldt A, Wetzel U, Lauschke J, et al. Fibrosis in left atrial tissue of patients with atrial fibrillation with and without underlying mitral valve disease. Heart 2004; 90: 400-5.

32. Polyakova V, Miyagawa S, Szalay Z, Risteli J, Kostin S. Atrial extracellular matrix remodeling in patients with atrial fibrillation. J Cell Mol Med 2008; 12: 189-208.

33. Bishop JE. Regulation of cardiovascular collagen deposition by mechanical forces. Mol Med Today 1998; 4: 69-75. (Review)

34. López B, González A, Querejeta R, Díez J. The use of collage-derived serum peptides for the clinical assessment of hypertensive heart disease. J Hypertens 2005; 23: 1445-51. (Review)

35. Nishikawa T, Edelstein D, Du XL, et al. Normalizing mitochondrial superoxide production blocks three pathways of hyperglycaemic damage. Nature 2000; 404: 787-90.

36. Brownlee M. Biochemistry and molecular cell biology of diabetic complications. Nature 2001; 414: 813-20.

37. Van Heerebeek L, Hamdani N, Handoko ML, et al. Diastolic stiffness of the failing diabetic heart: importance of fibrosis, advanced glycation end products, and myocyte resting tension. Circulation 2008; 117: 43-51.

38. Mukherjee R, Herron AR, Lowry AS, et al. Selective induction of matrix metalloproteinases and tissue inhibitor of metalloproteinases in atrial and ventricular myocardium in patients with atrial fibrillation. Am J Cardiol 2006; 97: 532-7.

39. Kato T, Yamashita T, Sekiguchi A, et al. AGEs-RAGE system mediates atrial structural remodeling in the diabetic rat. J Cardiovasc Electrophysiol 2008; 19: 415-20.

40. Raposeiras-Roubín S, Rodiño-Janeiro BK, Grigorian-Shamagian L, et al. Evidence for a role of advanced glycation end products in atrial fibrillation. Int J Cardiol 2012; 157: 397-402.

41. Iwasaki YK, Nishida K, Kato T, Nattel S. Atrial fibrillation pathophysiology: implications for management. Circulation 2011; 124 : 2264-74. (Review)

42. Gu J, Liu X, Wang QX, et al. Angiotensin II increases CTGF expression via MAPKs/TGF- $\beta 1 /$ TRAF6 pathway in atrial fibroblasts. Exp Cell Res 2012; 318: 2105-15

43. Xiao HD, Fuchs S, Campbell DJ, et al. Mice with cardiac-restricted angiotensin-converting enzyme (ACE) have atrial enlargement, cardiac arrhythmia, and sudden death. Am J Pathol 2004; 165 : 1019-32.

44. He X, Gao X, Peng L, et al. Atrial fibrillation induces myocardial fibrosis through angiotensin II type 1 receptor-specific Arkadiamediated downregulation of Smad7. Circ Res 2011; 108: 164-75.

45. Khatib R, Joseph P, Briel M, Yusuf S, Healey J. Blockade of the renin-angiotensin-aldosterone system (RAAS) for primary prevention of non-valvular atrial fibrillation: a systematic review and meta analysis of randomized controlled trials. Int J Cardiol 2012; 165: 17-24. (Review)

46. Xu D, Murakoshi N, Igarashi M, et al. PPAR- $\gamma$ activator pioglitazone prevents age-related atrial fibrillation susceptibility by improving anti-oxidant capacity and reducing apoptosis in a rat model. J Cardiovasc Electrophysiol 2012; 23: 209-17.

47. Shimano M, Tsuji Y, Inden Y, et al. Pioglitazone, a peroxisome proliferator-activated receptor-gamma activator, attenuates atrial fibrosis and atrial fibrillation promotion in rabbits with congestive heart failure. Heart Rhythm 2008; 5: 451-9.

48. Toblli JE, Cao G, Giani JF, Angerosa M, Dominici FP, GonzalezCadavid NF. Antifibrotic effects of pioglitazone at low doses on the diabetic rat kidney are associated with the improvement of markers of cell turnover, tubular and endothelial integrity, and angiogenesis. Kidney Blood Press Res 2011; 34: 20-33.

49. Gumieniczek A, Hopkała H, Roliński J, Bojarska-Junak A. Interleukin- 6 and oxidative stress in plasma of alloxan-induced diabetic rabbits after pioglitazone treatment. Immunopharmacol Immunotoxicol 2006; 28: 81-91.

50. Kamiński KA, Szepietowska B, Bonda T, et al. CCN2 protein is an announcing marker for cardiac remodeling following STZ-induced moderate hyperglycemia in mice. Pharmacol Rep 2009; 61: 496-503.

51. Puddu A, Sanguineti R, Durante A, Viviani GL. Pioglitazone attenuates the detrimental effects of advanced glycation end-products in the pancreatic beta cell line HIT-T15. Regul Pept 2012; 177: 79-84.

52. Oz Gul, Tuncel E, Yilmaz Y, et al. Comparative effects of pioglitazone and rosiglitazone on plasma levels of soluble receptor for advanced glycation end products in type 2 diabetes mellitus patients. Metabolism 2010; 59: 64-9.

53. Li F, Cai Z, Chen F, et al. Pioglitazone attenuates progression of aortic valve calcification via down-regulating receptor for advanced glycation end products. Basic Res Cardiol 2012; 107: 306

54. Saiki A, Ohir Ma, Endo K, et al. Pioglitazone decrease plasma angiotensin II concentration in type 2 diabetes. J Atheroscler Thromb 2010; 17: 651-7.

55. Subramanian V, Golledge J, Ijaz T, Bruemmer D, Daugherty A. Pioglitazone-induced reductions in atherosclerosis occur via smooth muscle cell specific interaction with PPAR \{gamma $\}$. Circ Res 2010; 107: 953-8.

56. Gu J, Liu X, Wang QX, et al. Beneficial effects of pioglitazone on atrial structural and electrical remodeling in vitro cellular models. J Mol Cell Cardiol 2013; 65: 1-8.

57. Chao TF, Leu HB, Huang CC, et al. Thiazolidinediones can prevent new onset atrial fibrillation in patients with non-insulin dependent diabetes. Int J Cardiol 2012; 156: 199-202. 